\title{
Autoconfianza y expectativas de carrera profesional en los menores en función del género. El uso de la creatividad para determinar el modelo aspiracional
}

\section{Self-confidence and professional expectations in kids according to gender. The use of creativity in defining a career aspiration model}

\author{
NÚÑEZ-GÓMEZ, Patricia ${ }^{1}$ \\ RODRIGO-MARTÍN, Luis ${ }^{2}$ \\ RODRIGO-MARTÍN, Isabel ${ }^{3}$ \\ MAÑAS-VINIEGRA, Luis ${ }^{4}$
}

\section{Resumen}

El objetivo principal de esta investigación fue comprender cómo operan los roles de género y las construcciones socioculturales, y su repercusión en la construcción de representaciones de profesiones "femeninas/masculinas", al objeto de poder determinar momentos y acciones clave de actuación que permitan luchar contra los estereotipos de género y orientarlos hacia la consecución de sus metas mediante el empoderamiento. La metodología empleada fue de carácter mixto: cualitativa (focus friends, focus groups y ejercicios proyectivos) y encuestas adaptadas a la infancia.

Palabras clave: autoconfianza, modelos aspiracionales, expectativas profesionales, creatividad, género.

\begin{abstract}
The main objective of this research was to understand how gender roles and socio-cultural constructions operate, and their repercussion on the construction of representations of "female/male" professions, in order to determine key moments and actions that allow fighting against gender stereotypes and guide them towards achieving their goals through empowerment. The methodology used was of a mixed nature: qualitative (focus friends, focus groups, and projective exercises) and surveys adapted to the characteristics of childhood.
\end{abstract}

Key words: self-confidence, aspirational models, professional expectations, creativity, gender.

\section{Introducción}

Las expectativas de futuro de los menores dependen, en buena medida, de la autopercepción que el entorno social y personal les permita desarrollar. A lo largo de la historia, el género ha determinado las percepciones

\footnotetext{
${ }^{1}$ Profesora Titular y Directora. Departamento de Ciencias de la Comunicación Aplicada. Universidad Complutense de Madrid. España. Email: pnunezgo@ucm.es

2 Profesor Contratado, Doctor. Área de Comunicación Audiovisual y Publicidad. Universidad de Valladolid. España. Email: luis.rodrigo@uva.es

3 Profesora Contratada, Doctora. Área de Comunicación Audiovisual y Publicidad. Universidad de Valladolid. España. Email: isabel.rodrigo@uva.es

${ }^{4}$ Profesor Ayudante, Doctor. Departamento de Ciencias de la Comunicación Aplicada. Universidad Complutense de Madrid. España. Email: Imanas@ucm.es
} 
profesionales y ha creado estereotipos que, a base de reproducirse, terminaban por condicionar no solo las realidades profesionales de la infancia, sino incluso sus deseos (Hayes, Bligler y Weisgram, 2018).

La creatividad es considerada, en la investigación, no solo como una herramienta para diagnosticar los modelos aspiracionales y las autopercepciones, sino también como un recurso para trabajar en las proyecciones de futuro y poder determinar las características y atributos que los niños y niñas consideran más relevantes en las diferentes profesiones, posibilitando que se puedan desarrollar intervenciones educativas que no condicionen su futuro profesional (Turner y Albro, 2017).

La Psicología Social, como ciencia aplicada, sirve a diferentes ámbitos, como la Educación, la Sociología, la Antropología, el Arte, la Comunicación, y otras tantas áreas de conocimiento, aportando datos de interés sobre la conducta de los individuos (Rizo-García, 2006). En el caso concreto de este estudio sobre las preferencias profesionales, entendidas como un fenómeno social, podría aportar datos interesantes para conocer las causas y las consecuencias que las originan.

La Educación, como institución al servicio de la socialización de los ciudadanos, se ocupa del estudio de los intereses vocacionales y profesionales del alumnado con la finalidad de orientarles en sus futuros estudios, dirigidos a satisfacer sus motivaciones profesionales y desarrollar las competencias clave (Tintaya-Condori, 2016). Así, comienzan a surgir diferentes servicios de orientación vocacional, laboral y profesional, y empiezan a diseñarse diferentes pruebas psicométricas con el fin de obtener datos para realizar la tarea de orientación (Santana-Vega, 1990). En otro ámbito, esta tarea también interesa a las empresas que, desde sus servicios de recursos humanos y de selección de personal, aspiran a atraer el talento de futuros trabajadores que, por sus aptitudes y actitudes, se ajusten al perfil de cada puesto de trabajo (Gallardo-Gallardo, Thunnissen y Scullion, 2019).

Las preferencias profesionales han sido objeto de estudio desde que surge la Psicología como ciencia independiente. Así, encontramos los trabajos de Fryer (1931) y Fitch (1935), en los que aparece el término de preferencias vocacionales y el concepto de la elección profesional. Más tarde, aparece la teoría de Ginzberg et al. (1951), en la que el estudio de las preferencias profesionales aparece unido al concepto de los intereses. En este sentido, podemos afirmar que cada persona tiene gustos y preferencias sobre diversos trabajos $y$ actividades. Mientras que algunas personas prefieren realizar acciones de ayuda a los demás, otras muestran preferencia por las tareas manuales o por el trabajo con máquinas, mientras que a otras personas les pueden gustar más las actividades relacionadas con el mundo del arte, el mundo científico, etc.

Para poder identificar los intereses profesionales, hay que tener claros tres conceptos diferentes (Soprano, 2013):

- Vocación: Es la llamada que surge desde el interior de cada uno, que le dirige a ejercer determinadas actividades.

- Ocupación: Es el lugar que ocupará una persona en el mundo, o el sitio que deberá construir para ejercer lo que tanto le gusta.

- Profesión: Es la conexión que permite fusionar la vocación con la ocupación, aquella especialidad que permite al individuo llevar a cabo las actividades que le gustan en el momento exacto, en el lugar propicio y el entorno adecuado.

Existen muchas definiciones de preferencias profesionales. Para Darley y Hagenah (1955), Super (1967) y Holland (1976), corresponde a la formulación explícita que hace una persona de su grado de atracción por una o varias actividades profesionales que reflejan las características de la personalidad y una fuente motivacional. Rivas (1990) añade que las preferencias profesionales son los mejores indicadores del desarrollo vocacional y que 
servirán para la orientación del alumnado hacia el mercado laboral. González Maura (2004) afirma que las preferencias profesionales son determinantes del desarrollo vocacional profesional de los estudiantes.

El interés por las preferencias profesionales se refleja en la obra de Rocabert (1987), quien las considera como un elemento clave de diagnóstico en la elección vocacional. Marín, Infante y Troyano (2000) señalan su valor predictor. En esta misma línea, encontramos los trabajos de Latiesa (1986), Gil Beltrán (2005) o Molina y Fernández (2007).

Las preferencias profesionales y su consecuente elección profesional deberían ser un acto de libertad individual, determinado por la motivación, aptitud, interés y conocimiento, pero, en realidad, esta decisión se ve condicionada por diferentes determinantes personales y contextos sociales que dirigen la decisión, tal y como apunta Álvarez (1995).

Uno de los determinantes que han condicionado, y aún sigue influyendo de manera importante en las elecciones profesionales, es el género, tanto el biológico como el social. Un estudio de la Fundación de Ayuda a la Drogradicción (FAD, 2007) afirma que en las preferencias profesionales de los jóvenes españoles de 15 a 24 años influye de manera decisiva el género, funcionando los estereotipos tradicionales con más fuerza de la que podríamos pensar en primera instancia. En este sentido, Grove (1983) reafirma la diferencia entre hombres y mujeres en relación a las preferencias profesionales.

La transferencia intergeneracional de normas con brecha de género ha incidido directamente en la brecha de las tasas de empleo, incluso en las sociedades más igualitarias (Haaland et al., 2018). Los estudios ambientales y de desarrollo confirman que la brecha de género existe en las expectativas masculinas y femeninas desde la socialización de la infancia, que supone un sesgo que crece en edades adultas, tanto en las profesiones desempeñadas como en los salarios (Solbes-Canales, Valverde-Montesino y Herranz-Hernández, 2020). La educación recibida al respecto durante la infancia es el mayor predictor sobre el éxito socioeconómico del adulto, tanto de su estado ocupacional como de los ingresos que obtendrá por su profesión, que en muchas ocasiones se ven mermados por los factores estructurales de la brecha de género por el trabajo parcial y la maternidad (Becker et al., 2019).

Vence, Palomares y Sánchez (2000) señalan que hay que considerar el efecto ejercido no solo por la variable sexo, sino también por la variable de identidad social de género. Existen otros determinantes que también condicionan las preferencias profesionales, entre los que se encuentran los denominados valores sociales; entre otros, González Maura (2004) y Rivas (2007) señalan el poder, el éxito social y ganar dinero. En muchas ocasiones, las elecciones vienen determinadas por estos valores sociales, seguidos de la vocación (Riart, 2001).

Por todo ello, el presente estudio pretende descubrir la evolución y persistencia de esa brecha de género, al objeto de poder realizar un mapa de las preferencias profesionales y de los modelos aspiracionales de los niños/as en España, en el momento actual. Además de las preferencias profesionales, esta investigación se centra en el análisis de las manifestaciones creativas de los sujetos infantiles que componen la muestra, concretadas en cómo se ven ellos realizando un trabajo y el contexto social en el que se produce. Para ello, se emplean diferentes modelos creativos apoyados por teorías como la Psicoanalítica (Kris, 1952; Kubie, 1956), de la que se obtendrá la descripción y la inspiración; la teoría Gestáltica (Wertheimer, 1959; Kohler, 1969), que aportará información sobre la percepción y la importancia de la figura fondo; la teoría Asociacionista (Mednick, 1962; Wallach, 1970), con la que se podrán analizar las relaciones y combinaciones que se establecen; la teoría Humanística (Rogers, 1959; Maslow, 1983), en la que se establecerán las necesidades y sus diferentes tipos, así como la autorrealización personal; la teoría Analítico-Asociativa (Hadamard, 1945; Koestler, 1964), relacionada con el descubrimiento de nuevas ideas y la posibilidad de descubrir nuevas soluciones. En este mismo sentido, 
la teoría Gestáltica-Asociativa (Gruber, 1974) nos acerca a la reestructuración del universo cognitivo, enriquecido con nuevas ideas.

El estudio de las preferencias profesionales del alumnado de Educación Primaria, el análisis de las creatividades plásticas realizadas por el alumnado y las diferencias de sexo y/o género social constituyen los tres grandes pilares sobre los que se sustenta el presente trabajo de investigación.

\subsection{Objetivos}

Los objetivos fundamentales de la presente investigación se vertebran en torno a dos grandes ejes. Por una parte, los relativos a los modelos aspiracionales y, por otra, los referentes al análisis de las percepciones profesionales.

Objetivos de los modelos aspiracionales:

- Determinar si los niños y las niñas desean parecerse a alguien en concreto cuando sean mayores, así como las causas y motivaciones de ese deseo.

- Explorar las percepciones de los/as niños/as sobre las semejanzas entre el modelo aspiracional y ellos mismos.

- Examinar las estrategias que van a seguir para alcanzar las metas aspiracionales descritas por ellos mismos respondiendo a la pregunta "¿Qué vas a hacer para parecerte al modelo aspiracional?"

Objetivos de las percepciones profesionales:

- Determinar cuáles son las profesiones más deseadas por los niños.

- Explorar las percepciones de esas profesiones que albergan en la infancia.

- Identificar los rasgos mejor y peor valorados de las profesiones y el grado de dificultad percibido para desempeñarlas.

- Examinar si la variable de género influye en las percepciones profesionales y si consideran que es un factor relevante, decisivo o, incluso, excluyente.

\section{Metodología}

Para llevar a cabo esta investigación, se utilizó una metodología de carácter mixto, con una primera fase de carácter cualitativo que permitió realizar una exploración significativa de las características de los sujetos de estudio y las posibles diferencias en cuanto a preferencias y aspiraciones en función del progreso evolutivo de los mismos, y una segunda fase, de carácter cuantitativo, que complementa y reafirma las impresiones sugeridas en la fase cualitativa. La utilización de esta metodología, compleja pero exhaustiva, permitió explorar en profundidad los objetivos descritos anteriormente.

Para ello, se formularon las siguientes preguntas de investigación que se reflejan en el cuadro 1.

En cuanto al análisis, se profundizó en el hecho de cómo el género condiciona que existan orientaciones hacia carreras que se consolidan o fortalecen desde pequeños. En otro nivel, se analizó cómo distintas variables como el género, religión, clase social, etc. influyen en sus proyecciones futuras. Se tomó como punto de partida la etapa psicoevolutiva del niño participante para utilizar las técnicas de investigación más adecuadas y conseguir los resultados óptimos. La duración y complejidad de las sesiones se adaptaron a esas etapas evolutivas, ya que es fundamental tomar en consideración que los niños disponen de una capacidad de atención y comprensión más limitada. En este sentido, cabe destacar que la observación y el dibujo se constituyeron como piedra angular 
del análisis y, para ello, en todas las sesiones, se optó por técnicas de estimulación de la expresividad, fundamentalmente a través del dibujo y el relato.

Cuadro 1

Preguntas de investigación

\begin{tabular}{|c|c|}
\hline Modelos aspiracionales & Precepciones profesionales \\
\hline $\begin{array}{l}\text { ¿Te gustaría parecerte a alguien de mayor? ¿Qué te gusta } \\
\text { de esa persona? } \\
\text { ¿Se parece en algo esa persona a ti? ¿En qué? } \\
\text { ¿Qué vas a hacer para parecerte a ella? }\end{array}$ & $\begin{array}{l}\text { ¿Qué profesiones te gustaría desempeñar en el futuro? } \\
\text { ¿Cómo imaginas esas profesiones? } \\
\text { ¿Qué consideras interesante de esa profesión? ¿Hay algo } \\
\text { que no te guste tanto? } \\
\text { ¿Crees que será difícil o fácil trabajar en eso? } \\
\text { ¿Cómo son las personas que se desempeñan en ese } \\
\text { trabajo?, ¿Qué cosas les gusta hacer? } \\
\text { ¿Es un chico o una chica? ¿Cuántos años tiene? } \\
\text { ¿Cómo es? } \\
\text { ¿Es una profesión que puede hacer cualquiera? ¿Quién } \\
\text { puede y quién no? }\end{array}$ \\
\hline
\end{tabular}

Fuente: Elaboración propia

Las técnicas utilizadas, descritas en el cuadro 2, se organizaron en torno a los focus friends, los focus groups y el ejercicio proyectivo denominado mi yo futuro.

\section{Cuadro 2}

Técnicas de investigación cualitativas

\begin{tabular}{|c|c|c|}
\hline Focus friends & Focus groups & Mi yo futuro \\
\hline $\begin{array}{l}\text { Sesiones cualitativas en las que se } \\
\text { trabajó con grupos pequeños -un } \\
\text { máximo de } 4 \text { participantes- para } \\
\text { garantizar su correcto } \\
\text { funcionamiento. } \\
\text { Son ideales para trabajar con edades } \\
\text { preescolares (4-5 años), ya que, al } \\
\text { estar en un entorno de confianza, se } \\
\text { expresan de forma más libre. } \\
\text { La duración fue de } 1 \text { hora } \\
\text { aproximadamente para evitar su } \\
\text { saturación y cansancio. } \\
\text { A lo largo de la sesión, se destinó } \\
\text { tiempo a realizar ejercicios libres } \\
\text { como dibujos o juegos de } \\
\text { asociaciones, siempre sin perder el } \\
\text { foco de estudio. }\end{array}$ & $\begin{array}{l}\text { Son sesiones formadas por un } \\
\text { máximo de } 6 \text { participantes y cuya } \\
\text { duración máxima fue de } 1,5 \text { horas. } \\
\text { Son perfectas para trabajar con niños } \\
\text { de edades superiores a } 6 \text { años, ya que } \\
\text { empiezan a alcanzar unos niveles de } \\
\text { expresividad y comprensión que } \\
\text { permiten trabajar en grupo. } \\
\text { Se intercalaron momentos de } \\
\text { discurso y momentos de descarga } \\
\text { para mantener sus niveles de energía } \\
\text { y atención a lo largo de toda la sesión. }\end{array}$ & $\begin{array}{l}\text { Se trata de un ejercicio proyectivo } \\
\text { que se realizó en todas las sesiones a } \\
\text { modo de cierre, en el que se solicitó a } \\
\text { los participantes que dibujasen cómo } \\
\text { se imaginan de mayores, haciendo } \\
\text { hincapié en su futura profesión. } \\
\text { Además, se escribieron cartas/notas a } \\
\text { sus yo futuros para entender cuáles } \\
\text { son sus dudas actuales. } \\
\text { Este ejercicio permitió completar el } \\
\text { análisis de sus discursos de un modo } \\
\text { más completo, al disponer de cada } \\
\text { uno de sus trabajos personales e } \\
\text { individuales. }\end{array}$ \\
\hline
\end{tabular}

Fuente: Elaboración propia

\subsection{Diseño de la muestra cualitativa}

Para llevar a cabo esta investigación cualitativa, se contó con un número total de 80 participantes, niños comprendidos entre las edades de 5-10 años, que se organizaron en tres intervalos de estudio:

- Kids 5-6 años, cursantes del último año de Educación Infantil.

- Kids 7-8 años, cursantes de 2을 de Educación Primaria.

- Kids 9-10 años, cursantes de 40 de Educación Primaria. 
Los grupos se segmentaron en función del género del participante para conseguir unas dinámicas más fluidas y homogéneas. Esta segmentación resulta especialmente interesante cuando los sujetos de estudio presentan edades tempranas, como es el caso, encontrándose inmersos en el proceso de construcción de su identidad.

En cuanto a la distribución geográfica del estudio cualitativo, fueron exploradas 3 zonas de investigación para alcanzar resultados representativos:

- Barcelona: perfil de familias emergentes.

- Madrid: perfil de familias emergentes, pero algo más normativas.

- Segovia: punto intermedio entre ambas.

La distribución de la muestra puede verse en el gráfico 1:

Gráfico 1

Diseño de la investigación cualtitativa

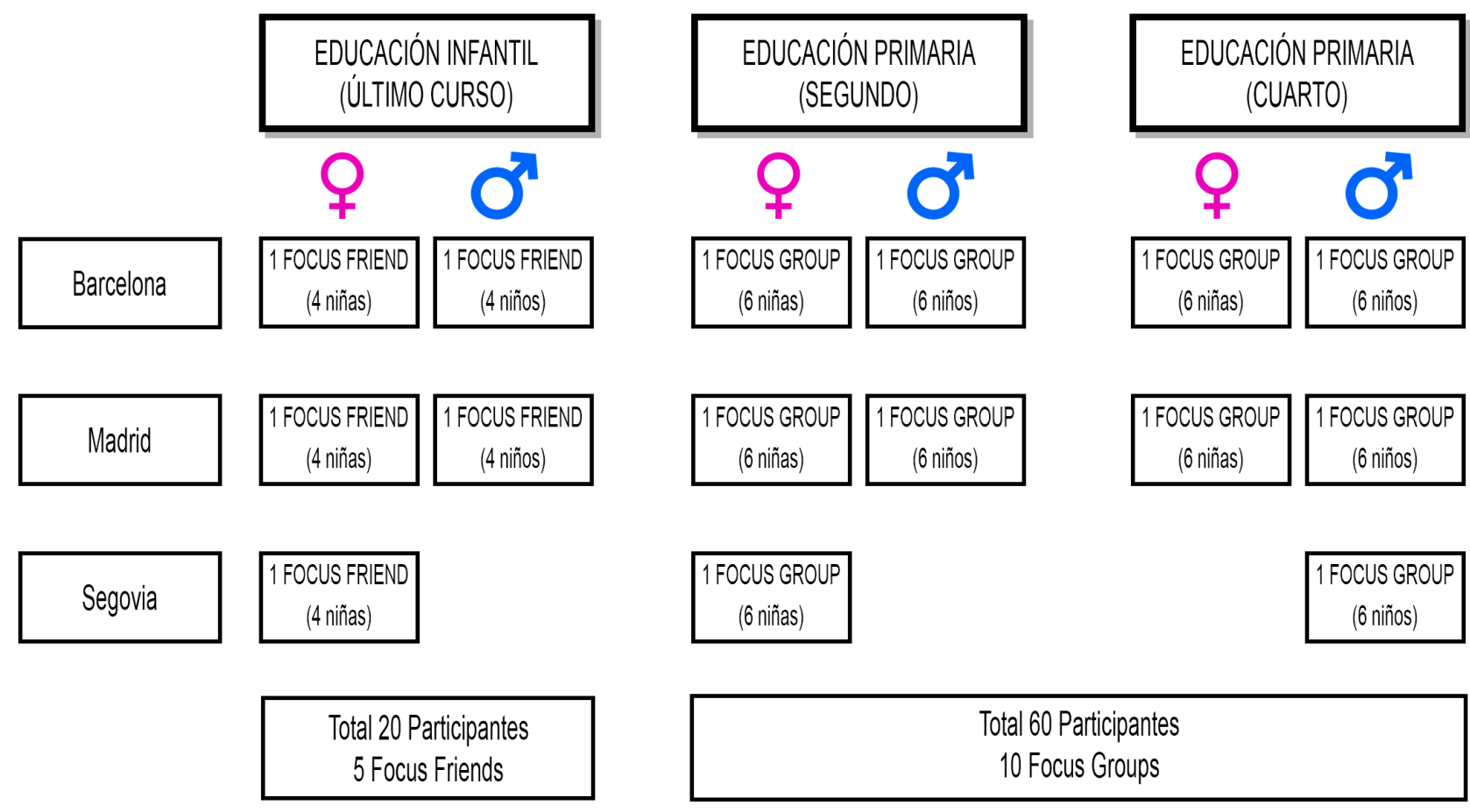

Fuente: Elaboración propia

\subsection{Diseño de la muestra cuantitativa}

Para el desarrollo de la fase cuantitativa, se administró un cuestionario con un total de 30 preguntas, validado y pre-testado con anterioridad con un porcentaje del $10 \%$ de la muestra.

El trabajo de campo se realizó en julio de 2019, con una muestra de 566 niños y niñas de siete a diez años (Generación Alpha), que, tras el cribado de validación, se redujo a 513 respuestas válidas. La muestra respeta los criterios de distribución geográfica de la parte cualitativa. La distribución en función del sexo de los cuestionarios realizados fue de 241 niñas $(46,98 \%)$ y 272 niños $(53,02 \%)$. Antes de esa edad es muy complicado que un niño, por sus características evolutivas, pueda rellenar por sí mismo un formulario, por lo que frecuentemente reciben ayuda de adultos que condicionan sus respuestas, incluyendo un sesgo importante que puede comprometer la investigación. En segundo lugar, el intervalo de 7 a 10 años permite estudiar los dos grupos de edades superiores que se han abordado en el estudio cualitativo. La distribución, supervisión y seguimiento de la encuesta fue 
realizada a través de la plataforma de investigación Qualtrics, empresa de estudios de mercado certificada por el código ICC/ESOMAR.

Para pertenecer a la muestra del estudio se requirió que los niños y las niñas encuestados fueran usuarios y usuarias de manera regular de un smartphone y/o tablet, y se contara, por motivos éticos, con la autorización y/o supervisión de su padre-madre y/o tutor, constando el correspondiente consentimiento informado y el informe favorable del Comité de Ética en Investigación del Departamento de Ciencias de la Comunicación Aplicada de la Universidad Complutense de Madrid. El intervalo de edades se determinó como el adecuado, por corresponder a los dos grupos de edades superiores de los empleados en la parte cualitativa, descartando los rangos de edades inferiores por la dificultad de trabajar con cuestionarios, por su escasa autonomía y para evitar influencias de los adultos en las respuestas. La distribución geográfica de la fase cuantitativa respetó las ciudades de Madrid, Barcelona y Segovia, para facilitar la comparativa de resultados.

Durante los meses de septiembre y noviembre de 2019, se realizó el tratamiento estadístico de los datos con base en las preguntas de investigación e hipótesis asociadas, usando para este fin el Software SPSS v.25.

\section{Resultados}

\section{1. ¿Cómo son los niños?}

Antes de poder dar respuesta a qué quieren ser de mayores los niños y por qué quieren desarrollar esas profesiones, debemos comprender los mecanismos por los que construyen proyecciones de futuro. Debemos acudir a la ciencia de la Psicología para poder encontrar las variables que influyen en el autoconcepto (FernandesSisto, Marín-Rueda y Urquijo, 2010; Ternera, 2014), tanto a nivel evolutivo como social. Debemos tener en consideración que el autoconcepto tiene un carácter multidimensional y evolutivo (Pérez, 1993; Esnaola, Goñi y Madariaga, 2008; Zabala y Palacios, 2008).

En esta línea, el autoconcepto es la percepción que el niño tiene de sí mismo, y está constituido por distintos aspectos que van transformándose a lo largo de la vida. En concreto, encontramos diferencias significativas en las dimensiones que forman el autoconcepto según la etapa evolutiva en la que se encuentra el kid. Debemos prestar atención a dos niveles: el evolutivo y el social. En cuanto al primero, encontramos que los niños en los que se centró el estudio encuentran sus referentes y modelos en familiares cercanos (hasta 6 años). A partir de esa edad, empiezan a abrir el prisma hacia amigos y personas ajenas a la familia, por lo que el autoconcepto se articula a partir de logros y fracasos. Comienza la formación de su identidad sexual y la noción de sexo a partir de los 6-8 años, aumentan sus interacciones sociales y la necesidad de aceptación social. En cuanto al segundo nivel, el social, encontramos que sus niveles de exposición a la tecnología no tienen precedentes, constituyen una generación fluida (on/offline indiferenciados), presentando un alto dominio tecnológico que les permite estar siempre conectados. Sus padres fueron millennials, por lo que recibieron una fórmula de crianza más participativa que generaciones anteriores; suelen ser críticos, realistas y pragmáticos, y desarrollan una alta responsabilidad medioambiental.

Estas características repercuten en un target altamente activo, que prefiere actividades de descarga física y en el que la tecnología está muy presente desde las edades más tempranas (4-5 años). Entre los grupos encontramos una serie de diferencias:

- En el segmento de 5-6 años: Las actividades más preponderantes son las que se desarrollan fuera del hogar, normalmente intervienen otros miembros de la familia o mascotas y suelen existir altos niveles de descarga de energía (correr, saltar, juegos físicos, deportes...). 
- En el segmento de 7-8 años: El juego físico continúa muy presente, pero irrumpe la tecnología a través de los videojuegos (fundamentalmente en niños) y cualquier actividad plástica (fundamentalmente entre las niñas).

- En el segmento de 9-10 años: Jugar y compartir con otros amigos toma un gran protagonismo en ambos sexos. Las niñas desarrollan comportamientos sociales en los que la presencia de unas con otras es fundamental, sin actividad concreta de por medio, mientras que en los niños el juego es preponderante, tanto on como offline.

Con base en el genero, se puede apreciar, especialmente en los dos segmentos de mayor edad, que las niñas expresan con mayor claridad las emociones que se desprenden de sus actividades favoritas ("bailar me gusta porque me siento alegre"-niña de 7-8 años); mientras tanto, los niños muestran un mayor interés en relacionarse con los amigos a través del juego ("estar jugando con los amigos a la Play, o en la calle"-niño de 9-10 años). A nivel de diferencias, de un modo espontáneo, entre los niños son más frecuentes discursos en los que se puntualizan aquellas actividades en las que resultan más competitivos o ganadores, en comparación con las niñas, que valoran más la aceptación grupal.

En cuanto a las actividades que ya no les resultan agradables, se aprecia que, de un modo transversal, destacan las actividades que están relacionadas con el cumplimiento de las normas, fundamentalmente las relacionadas con las obligaciones en la realización de sus tareas, las que se relacionan con edades anteriores y las que suponen una restricción en el acceso a las tecnologías. A partir de los 7 años, en ambos sexos empiezan a aparecer verbalizaciones frecuentes que hacen referencia a actividades que ya no les gustan porque se atribuyen al genero opuesto. Cabe recordar que, en estas edades, se encuentran en unas etapas en la que la definición del autoconcepto cobra fuerza, por lo que es el momento en el que se extreman las diferencias.

\subsection{Las familias}

El análisis de su entrono permite entender cómo este influye en la formación de su autoconcepto (Ponsa-Díez, 1998; García-Cruz, 2016) y en las diferencias de género que se fomentan desde edades tempranas (Calvo, González y Martorell, 2001; Etxebarría et al., 2003).

Las niñas verbalizan de un modo más directo y natural que reciben ayuda y apoyo emocional de sus padres, hecho que contribuye a un autoconcepto en el que se atribuye mayor peso a la expresión de las emociones. Por el contrario, los niños también se expresan en términos relacionados con las emociones en referencia a sus padres, pero tienden a puntualizar logros, lo cual repercutirá también en su definición futura. Pese a estas diferencias, en términos generales, la familia comparte significados globales basados en el amor, la felicidad, la alegría y la complicidad.

\subsection{Los amigos}

En este caso, los significados más compartidos -al preguntar por sus amigos- son diversión, juego y compañía, aunque existen notables diferencias entre los tres segmentos estudiados:

- En niños de 5-6 años: El grupo cercano de amigos en una de las principales fuentes de diversión y juego. Prácticamente no existen diferencias de género en sus definiciones, evaluando todos al grupo de amigos/as como muy positivo.

- En los niños de 7-8 años: Las relaciones con el grupo empiezan a ser más complejas y aparecen tensiones entre los grupos. Entre las niñas se hacen más notables las diferenciaciones entre amigos y amigas, mostrando cierta polarización.

- En el segmento de 9-10 años: Son el grupo de apoyo y confidente más importante y se aprecia un progresivo protagonismo y desplazamiento del referente que anteriormente ocupaban los padres. 


\subsection{Autopercepción, ¿cómo son los kids?}

Todos los aspectos analizados anteriormente repercuten de un modo directo e indirecto en la propia definición que un niño hace de sí mismo. Se definen a través de diversas dimensiones, que adquieren más peso en función del sexo estudiado. Por un lado, las niñas hacen mayor referencia a aquellas características relacionadas con la expresividad de aspectos de personalidad que les atribuyen sus padres. Del mismo modo, entre las más pequeñas (hasta 9 años) se refieren a ellas mismas en términos de belleza o rasgos físicos positivos y que están bien aceptados entre la sociedad. Por otro lado, los niños hablan de ellos mismos en términos competitivos/ganadores y se mueven en un espectro emocional relacionado con la adrenalina y la peligrosidad. La observación de los focus groups crea una diferencia significativa entre niños y niñas. Mientras que los discursos de los niños giran en torno a la competitividad y la fuerza, el discurso que mantienen las niñas crea un marcador diferente, como es la preocupación por el aspecto físico, algo que prácticamente no se encuentra en los focus groups del género opuesto. Para poder trazar una descripción de cómo se ven los niños, se deben contemplar varios aspectos:

- Logros y habilidades: Es un primer nivel de definición común a todas las edades y sexos, en el que se describen a partir de aquellas cosas que saben hacer muy bien o de las que se sienten orgullosos. Tienden a ser habilidades principalmente físicas y, en segundo lugar, artísticas.

- Aspecto físico: Es una dimensión presente principalmente entre las niñas de 5-8 años, y que no aparece (o es muy débil) entre los niños. En este sentido, las niñas se definen a partir de características que sus progenitores les valoran como positivas, generando así una apropiación de su discurso.

- Características emocionales: Se trata también de una dimensión más presente en la autodefinición de las niñas. Hace referencia a características de personalidad (también socialmente bien aceptadas y positivas). Estos rasgos aparecen ligeramente entre los niños a partir de los 8 años.

- Competición: Es una dimensión presente fundamentalmente en los niños, que se puede apreciar ligeramente entre las niñas de 8-10 años, y que habla de ellos en términos ganadores.

Gráficamente, puede realizarse la siguiente radiografía del autoconcepto de niños y niñas de la siguiente manera (cuadros 3 y 4 ):

\section{Cuadro 3}

Así soy Yo: definición de las niñas

\begin{tabular}{|c|c|c|c|}
\hline Logros y habilidades & Aspecto físico & Competición & Características emocionales \\
\hline $\begin{array}{l}\text { Yo soy guay [muy bueno, } \\
\text { interesante] dibujando (5-6 } \\
\text { años). } \\
\text { Yo sé hacer el pino } \\
\text { [ejercicio físico] (5-6 años). } \\
\text { A mí se me da bien el salto } \\
\text { hípico y también hago } \\
\text { gimnasia rítmica y ballet (7- } \\
8 \text { años). } \\
\text { A mí me gusta, como a las } \\
\text { niñas de mi clase, saber } \\
\text { hacer muchas cosas, } \\
\text { abrirme de piernas en } \\
\text { gimnasia (7-8 años). } \\
\text { Yo las multiplicaciones me } \\
\text { las sé todas (9-10 años). }\end{array}$ & $\begin{array}{l}\text { Yo soy muy guapa (5-6 } \\
\text { años). } \\
\text { Me gusta ser guapa (5-6 } \\
\text { años). } \\
\text { Me gusta que soy bonita } \\
\text { para mí misma, me gusta } \\
\text { verme en el espejo (7-8 } \\
\text { años). }\end{array}$ & $\begin{array}{l}\text { A mí me gusta el kung fu, } \\
\text { porque mi hermano hace } \\
\text { aikido; si la gente me hace } \\
\text { daño, me puedo defender } \\
\text { (7-8 años). } \\
\text { A mí me dijeron que soy } \\
\text { buena nadando y gano } \\
\text { competiciones nacionales } \\
\text { (9-10 años). }\end{array}$ & $\begin{array}{l}\text { Me gusta ser guapa, alta y } \\
\text { simpática (5-6 años). } \\
\text { Guapa, simpática y ser } \\
\text { presumida (5-6 años). } \\
\text { A mí me gusta que ayudo a } \\
\text { los demás (7-8 años). } \\
\text { No sé lo que más me gusta } \\
\text { de mí misma, pero sí que lo } \\
\text { saben mis amigos, que } \\
\text { estoy muy loca (7-8 años). } \\
\text { Yo diría que soy un poco } \\
\text { despistada y de conversar y } \\
\text { hablar con mis amigos (9- } \\
10 \text { años). } \\
\text { Mi madre a mí me dice que } \\
\text { a veces soy muy brusca... y } \\
\text { luego la gente dice que soy } \\
\text { muy buena y educada (9-10 } \\
\text { años). }\end{array}$ \\
\hline
\end{tabular}

Fuente: Elaboración propia 


\section{Cuadro 4}

Así soy Yo: definición de los niños

\begin{tabular}{|c|c|c|c|}
\hline Logros y habilidades & Aspecto físico & Competición & Características emocionales \\
\hline $\begin{array}{l}\text { Yo, la voltereta y el pino } \\
\text { [ejercicio físico] (5-6 años). } \\
\text { Soy bueno en el Fornite (5- } \\
6 \text { años). } \\
\text { Lo mejor que hago es tirar } \\
\text { con arco ( } 7-8 \text { años). } \\
\text { Soy bueno en ser fuerte, } \\
\text { rápido y ganar (9-10 años). }\end{array}$ & $\begin{array}{c}\text { No surgen respuestas al } \\
\text { respecto. }\end{array}$ & $\begin{array}{l}\text { Yo soy el más rápido de } \\
\text { toda mi familia (5-6 años). } \\
\text { Yo soy el más mega [el } \\
\text { mejor] de todos (5-6 años). } \\
\text { Sé competir en torneos de } \\
\text { ajedrez (7-8 años). } \\
\text { Yo sé mucho de Fornite (9- } \\
10 \text { años). } \\
\text { A mí me gustan mucho las } \\
\text { emociones fuertes en } \\
\text { cuanto a montañas rusas } \\
\text { (9-10 años). } \\
\text { Antes era menos } \\
\text { competitivo en los } \\
\text { videojuegos y ahora } \\
\text { siempre quiero ser el mejor } \\
\text { de la clase (9-10 años). } \\
\text { Corro mucho, gano muchos } \\
\text { trofeos, soy muy bueno en } \\
\text { la portería y soy muy fuerte } \\
\text { (9-10 años). }\end{array}$ & $\begin{array}{l}\text { A mí, que hago caso a mi } \\
\text { madre, y a mi abuela, } \\
\text { portarse bien más o menos } \\
\text { (9-10 años). } \\
\text { Que soy listo, amable y } \\
\text { comparto ( } 9-10 \text { años). } \\
\text { Yo antes me portaba un } \\
\text { poco mal y ahora me porto } \\
\text { mejor ( } 9-10 \text { años). }\end{array}$ \\
\hline
\end{tabular}

Fuente: Elaboración propia

\section{5. ¿Cómo se perciben a sí mismos en el futuro?}

En la descripción del Yo futuro que contempla la investigación, se amplían las definiciones del autoconcepto presente, incorporando dos grandes variables que toman relevancia en su discurso. Por una parte, el trabajo, presente en la totalidad de las respuestas de los niños participantes; y es precisamente a partir del trabajo, que se imaginan realizando en el futuro, de donde surgen la mayor parte de sus descripciones. No se aprecian diferencias significativas, ni en función de género, ni de edad. Por otra parte, se identifica la variable familia, al igual que con el trabajo constante y presente en todas las respuestas. La gran mayoría de los niños que conforman la muestra se proyectan teniendo familia, hijos y rodeados de sus mejores amigos actuales. Sin embargo, el estudio de esta variable sí arroja diferencias significativas por género, en tanto que las niñas tienen incorporado en su discurso el hecho de querer ser madres e, incluso, algunas se proyectan siendo esencialmente madres.

\subsection{Diferencias en base al género}

En cuanto a las diferencias en sus proyecciones de futuro, se identifican dos dimensiones que adquieren significados distintos con base en el género:

- El físico: Cuando hablan de su Yo futuro, el físico es un elemento importante, especialmente en edades inferiores a los 8 años. A nivel de diferencias, sí identifica que, mientras las niñas refuerzan características relacionadas con la belleza, los niños lo hacen en relación a la fuerza/potencia. Por ejemplo: "Seré una profesora guapa, muy buena, un poco regañona, con pendientes que cuelgan y bolso" (niña 5-6 años); "me imagino alto y fuerte, como Hulk" (niño 5-6 años).

- El esfuerzo y las normas: En el discurso de las niñas se observan relatos más enfocados hacia el comportamiento normativo: "cuando sea mayor tendré que ir al instituto" (niña 5-6 años), "podremos ir 
[a la discoteca] y ellos no nos lo van a poder prohibir" (niña 7-8 años). Por otro lado, entre los niños se aprecian discursos en los que se proyecta el futuro con base en el éxito: "ser el máximo goleador, eso sería lo mejor" (niño 7-8 años).

Como es natural, de sus proyecciones de futuro se desprenden dudas en relación con su vida adulta. En todas ellas se aprecia que el discurso de las niñas alberga mayores preocupaciones en relación a si se ha conseguido su pretensión y cómo ha sido el proceso. En lo referente a la familia, tanto niñas como niños están interesados en saber cómo será su familia y si tendrán hijos/as. Sin embargo, en el caso de las primeras, se muestra mucha mayor preocupación por saber cómo estarán los miembros de su familia y las amigas actuales. En lo referente al trabajo, existe una preocupación compartida por ambos sexos, pero en el caso de las niñas el interés se acentúa por saber si les ha costado mucho esfuerzo conseguirlo (especialmente niñas de más de 8 años) y si les agrada o no el desempeño profesional. Finalmente, en cuanto al aspecto físico, cabe destacar que es una variable a la que otorgan menor importancia los niños y que pierde importancia en las niñas al superar la edad de 9 años.

\subsection{Referentes}

El estudio arroja diferencias significativas en cuanto a los referentes de los kids, fundamentalmente en función de la edad y no tanto del sexo.

En el tramo de 5-6 años, los referentes inmediatos son aquellos del entorno más cercano, y con los que se comunican en persona en su día a día. Los padres/madres son los referentes inmediatos y principales, posicionándose como el máximo ejemplo de sus conductas, influencia que se proyecta en algunos casos a los abuelos/abuelas. Los profesores también son un modelo muy presente en ellos, y de sus respuestas se desprende cariño y cierta admiración. Finalmente, los amigos, que se presentan como modelos de éxito y de los que destacan tanto sus habilidades como sus características físicas.

En cuanto al segundo segmento estudiado, entre 8-10 años, se aprecia que aparece una nueva dimensión relacionada con la irrupción e importancia de personajes famosos, como cantantes, actrices y deportistas mediáticos. Los principales motivos que tienen para querer parecerse a ellos giran en torno a los modelos de éxito que promueven y a su aspecto físico, muy relacionado con la aceptación social. La aparición de este nuevo referente está muy vinculado con el acceso de los kids a Internet. A nivel de género, no se aprecian diferencias significativas en relación con los modelos aspiracionales elegidos. Si bien las niñas hacen más hincapié en la belleza, para ambos sexos es importante que sea un perfil de éxito que triunfe en su profesión.

\section{8. ¿Qué van a ser de mayores? Las profesiones}

Después del ejercicio de proyección sobre su futuro, se exploraron las aspiraciones laborales de los sujetos de estudio respecto a su futuro. A nivel general, se observaron profesiones diversas que se distribuían entre la fantasía (ser princesa o superhéroe) a otras más realistas. Al profundizar en los motivos, se aprecia que, en su mayoría, se relacionan con la imitación de sus referentes más cercanos. Entre los referentes se encuentran modelos familiares, de la escuela y de los medios de comunicación. Además, el juego y el refuerzo de ciertas habilidades cobra relevancia a la hora de idear su futuro laboral. Se detecta un abanico de posibilidades, en el que pueden destacarse algunas categorías de carácter transversal que adquieren diferente peso en función de la edad y el género. En un primer nivel (cuadro 5), exponen preferencias profesionales relacionadas con su vida cotidiana, presentes en su día a día y, principalmente, con el entorno escolar: 


\section{Cuadro 5}

Primer nivel aspiracional

\begin{tabular}{|l|l|l|l|}
\hline \multicolumn{1}{|c|}{ Educación } & \multicolumn{1}{|c|}{ Sanidad } & \multicolumn{1}{c|}{ Ciencias / Ingeniería } & \multicolumn{1}{c|}{ Deporte } \\
\hline En conjunto, es la & Las profesiones & Las profesiones & Es una categoría más \\
categoría más & sanitarias también tienen & científicas están muy & presente entre los niños. \\
mencionada entre las & un peso relevante en las & presentes en las & Engloba profesiones en \\
niñas. Engloba diferentes & aspiraciones de las niñas. & aspiraciones de los & las que el deporte de \\
profesiones relacionadas & Ser médico/veterinario & niños. No se observan & competición es el \\
con la enseñanza. & es la profesión más & diferencias en función & principal protagonista. \\
& aspirada dentro de la & del género. & \\
& categoría. & & \\
\hline
\end{tabular}

Fuente: Elaboración propia

En un segundo nivel (cuadro 6), se encuentran profesiones que están más relacionadas con los hobbies y las actividades de preferencia en la infancia:

Cuadro 6

Segundo nivel aspiracional

\begin{tabular}{|c|c|c|c|}
\hline Artistas & Seguridad & Dibujo & Piloto \\
\hline $\begin{array}{l}\text { Se trata de una categoría } \\
\text { con algo más de peso } \\
\text { entre las niñas y que } \\
\text { comprende profesiones } \\
\text { relacionadas con el } \\
\text { canto, el baile, la } \\
\text { interpretación o ser } \\
\text { YouTuber. }\end{array}$ & $\begin{array}{l}\text { Categoría de preferencia } \\
\text { fundamentalmente } \\
\text { masculina y abarca todas } \\
\text { las profesiones en las } \\
\text { que se protege de algún } \\
\text { modo a un tercero, como } \\
\text { policías, socorristas o } \\
\text { militares. }\end{array}$ & $\begin{array}{l}\text { Es una categoría con } \\
\text { presencia similar en } \\
\text { ambos sexos y que } \\
\text { presenta profesiones } \\
\text { como dibujante, } \\
\text { diseñador de } \\
\text { videojuegos, pintor o } \\
\text { escultor. }\end{array}$ & $\begin{array}{l}\text { Se relaciona } \\
\text { exclusivamente con } \\
\text { niños e implica conducir } \\
\text { aviones, coches, barcos, } \\
\text { motos o cohetes } \\
\text { espaciales. }\end{array}$ \\
\hline
\end{tabular}

Fuente: Elaboración propia

Existe un tercer nivel, en el que se relacionan profesiones dispersas en función de las que desempeñan sus referentes más directos. Entre las niñas destacan ser limpiadora, peluquera y, muy significativamente, ser mamá $y$, entre los niños, dependiente y camarero.

En un análisis relacionado con el género (gráfico 2), comprobamos cómo las categorías más mencionadas se corresponden en su mayoría con los atributos y estereotipos tradicionalmente asociados a los roles femeninos y masculinos. En conjunto, los niños eligen profesiones relacionadas con la fortaleza y la adrenalina, y las niñas se decantan por profesiones vinculadas al cuidado ajeno y al arte. Cabe destacar que las profesiones relacionadas con la ciencia y la ingeniería constituyen una aspiración de género neutro y que en las edades objeto de estudio son elegidas de igual manera por niños y niñas. 


\section{Gráfico 2}

Distribución por género de las preferencias aspiracionales

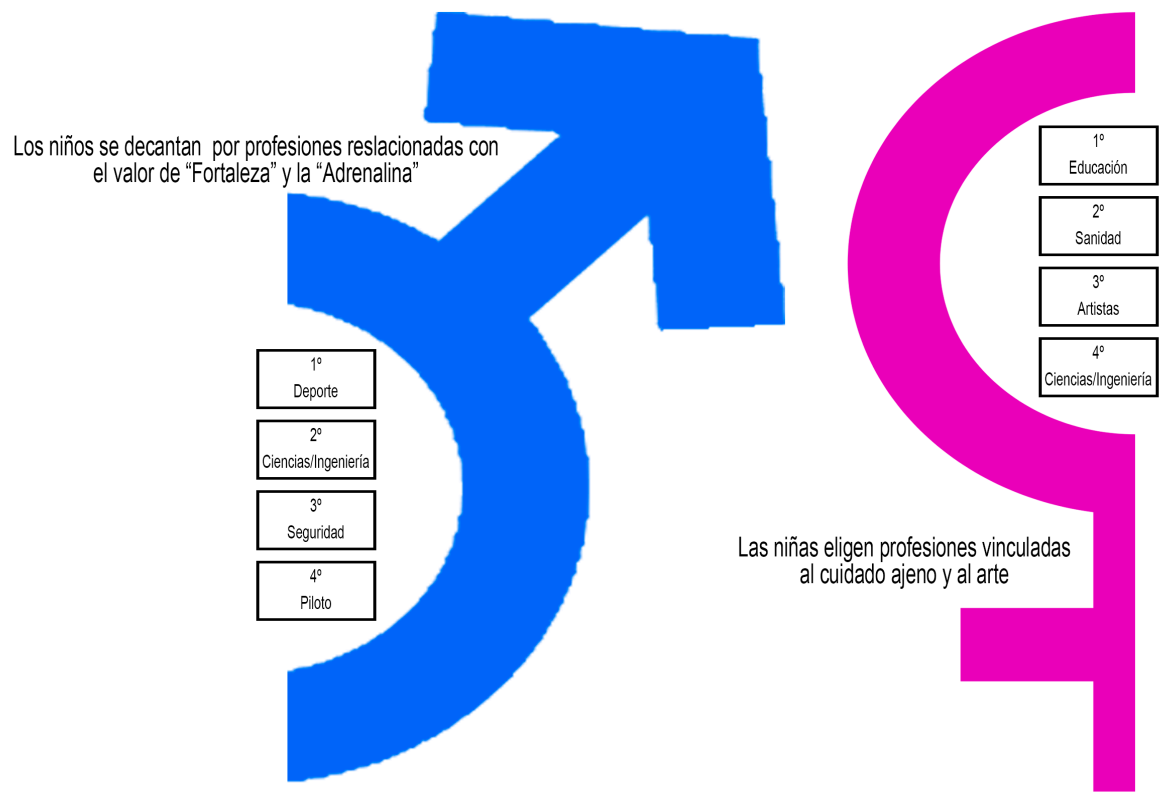

Fuente: Elaboración propia

Como puede apreciarse, los niños eligen profesiones principalmente relacionadas con la fuerza física. La correlación de preferencias es la siguiente:

- En primer lugar, el deporte: Es la profesión que mas interés genera y en la que destacan los modelos aspiracionales más alejados de la familia y la escuela.

- En segundo lugar, la ciencia y la ingeniería: Se observa una fuerte aspiración hacia la ciencia y la exploración del mundo que les rodea, algo que concuerda con el momento evolutivo que les corresponde por edad.

- En tercer lugar, la seguridad: Es una categoría destacada en los niños, porque se asocia a la aventura, la fuerza y la protección.

- En cuarto lugar, piloto: Destaca esta elección por lo que conlleva a nivel emocional (retos) y racional/material (implica conducir un artefacto).

En cuanto a las elecciones de las niñas, el estudio arroja la conclusión de que el cuidado de la persona ajena, especialmente hacia los niños, tiene una enorme preponderancia. Además, también aspiran a trabajar en el arte y la ciencia:

- En primer lugar, la educación: Las niñas, en su mayoría, se decantan por profesiones relacionadas con el bienestar de los más pequeños. La profesión mas mencionada es la de maestra/profesora.

- En segundo lugar, la sanidad: Las profesiones sanitarias también presentan niveles importantes en las aspiraciones de las niñas.

- En tercer lugar, el arte: Las proyecciones en el terreno artístico tiene que ver con la existencia de hobbies relacionados con actividades artísticas como cantar, bailar, dibujar...

- Finalmente, la ciencia y la ingeniería: Al igual que sucede en los niños, el momento evolutivo invita a explorar el mundo que les rodea y la ciencia invita a ello. 


\subsection{Resultados cuantitativos}

Los resultados de la parte cuantitativa muestran que los resultados obtenidos cualitativamente se reproducen con pequeñas variaciones, con la ventaja de que pueden cuantificarse, por lo que puede determinarse que las preferencias de niños y niñas se distribuyen de la siguiente manera (cuadro 7):

Cuadro 7

Distribución de la aspiración profesional en función del género obtenido en la fase cuantitativa

\begin{tabular}{|l|r|l|r|}
\hline \multicolumn{2}{|c|}{ Niños } & \multicolumn{2}{c|}{ Niñas } \\
\hline Seguridad & $28,8 \%$ & Sanidad & $37,4 \%$ \\
\hline Sanidad & $26,5 \%$ & Educación & $29,2 \%$ \\
\hline Deporte & $26,3 \%$ & Arte & $16,3 \%$ \\
\hline Ciencia & $11,0 \%$ & Ciencia & $10,5 \%$ \\
\hline Otros & $\mathbf{7 , 4 \%}$ & Otros & $6,6 \%$ \\
\hline Total & $\mathbf{1 0 0 , 0 \%}$ & Total & $\mathbf{1 0 0 , 0 \%}$ \\
\hline
\end{tabular}

Fuente: Elaboración propia

De este modo, la seguridad, la sanidad y el deporte encabezan las preferencias de los niños, mientras que la sanidad y la educación destacan en los resultados obtenidos en las niñas. Hay que considerar que, a pesar de estas pequeñas variaciones que se producen entre la investigación cualitativa y la cuantitativa, esta última proporciona resultados más conscientes, más meditados y que, por tanto, existe una mayor influencia de factores ajenos a las aspiraciones reales de los propios niños, como, entre otros, lo que los demás esperan de ellos.

\section{Conclusiones}

A lo largo del estudio se ha podido comprobar la importancia del conocimiento de las profesiones para generar expectativas y vocaciones en los niños. En todos los grupos de edades, pero especialmente en los más pequeños, el modelo aspiracional se construye fundamentalmente por referencia a sus seres más cercanos, fundamentalmente los miembros de su familia, bien sea por querer repetir las profesiones de sus familiares, o por reproducir los consejos que esos familiares les ofrecen en cuanto a la decisión sobre qué profesión es mejor para ellos en el futuro, por lo que el factor de imitación o admiración tiene un peso absolutamente relevante, que progresivamente se va desplazando desde el grupo primario de la familia a otros grupos de carácter más social, como los amigos.

En el estudio de la creatividad se ha podido analizar la relación necesaria entre la autoconfianza y el modelo de expectativa de carrera profesional. En todos los grupos estudiados se identifica que los niños y niñas se ven a sí mismos como perfectamente cualificados y capacitados para el desempeño de las tareas profesionales elegidas.

En cuanto a su visión sobre las exigencias para alcanzar el nivel competencial que exigen las profesiones a las que aspiran, parece que no hay un pensamiento suficientemente elaborado, ya que la mayoría de los sujetos, sin distinción de género, piensan que pueden conseguirlo sin demasiados problemas, y solo en algunas ocasiones se limitan a repetir un discurso escuchado por los adultos, como el de "hay que estudiar mucho", pero sin generar una sensación de dificultad. 
En cuanto a las preferencias profesionales, se ha podido determinar que la variable de género influye de manera efectiva. Mientras que los niños prefieren profesiones relacionadas con la seguridad, el orden o la destreza deportiva, que exigen un desarrollo más relacionado con las destrezas físicas, las niñas eligen profesiones más relacionadas con el cuidado y protección de los demás. El deporte-fuerza, velocidad o resistencia- es la opción en la que más se manifiesta la brecha de género entre los niños y las profesiones artísticas -sensibilidad, estética o esmero-, entre las niñas. Sin embargo, el género no es una variable que influya en la preferencia por la ciencia -última elección-

Como conclusión final, puede afirmarse que las preferencias de niños y niñas en cuanto a carreras profesionales y expectativas de consecución de las mismas continúan teniendo un sesgo en función del género, si bien es cierto que esa diferencia va menguando y resulta prácticamente inexistente en algunas categorías profesionales, especialmente la ciencia. La importancia de los referentes aspiracionales resulta esencial a la hora de determinar las preferencias en la infancia. Por todas estas razones, las políticas de igualdad que favorezcan el empoderamiento, el desarrollo de la creatividad y la visibilidad de los logros de personas adultas suponen una necesidad de primer orden en el contexto sociocultural actual para conseguir una progresiva disminución de las desigualdades y la ruptura de la brecha de género en el desempeño profesional.

\section{Financiación}

Esta investigación ha sido financiada por el Proyecto Art. 83 LOU no 516-2019, celebrado entre la Universidad Complutense de Madrid y Mattel España, titulado Self-confidence and career expectations in the kids depending of the gender, dentro de las actividades llevadas a cabo en la Cátedra Extraordinaria de Comunicación Digital de Infancia y Adolescencia de la Universidad Complutense de Madrid.

\section{Referencias bibliográficas}

Álvarez, M. (1995). Orientación profesional. CEDESEC.

Becker, M., Baumert, J., Tetzner, J., Maaz, K., \& Koller, O. (2019). Childhood Intelligence, Family Background, and Gender as Drivers of Socioeconomic Success: The Mediating Role of Education. Developmental Psychology, 55(10), 2231-2248. https://doi.org/10.1037/dev0000766

Calvo, A. J., González, R., \& Martorell, M. C. (2001). Variables relacionadas con la conducta prosocial en la infancia y adolescencia: personalidad, autoconcepto y género. Infancia y aprendizaje, 24(1), 95-111. https://doi.org/10.1174/021037001316899947

Darley, J., \& Hagenah, T. (1955). Vocational Interest Measurement: Theory and Practice. University of Minnesota Press.

Esnaola, I., Goñi, A., \& Madariaga, J. M. (2008). El autoconcepto: perspectivas de investigación. Revista de psicodidáctica, 13(1), 69-96. https://bit.ly/3g6x0Sv

Etxebarría, I., Apodaca, P., Eceiza, A., Fuentes, M. J., \& Ortiz, M. J. (2003). Diferencias de género en emociones y en conducta social en la edad escolar. Infancia y aprendizaje, 26(2), 147-161.

https://doi.org/10.1174/021037003321827759

$\operatorname{FAD}(2007,12$ de septiembre). Valores y preferencias profesionales de los jóvenes españoles de 15 a 24 años. Comunidad Escolar no 815. Periódico digital de Información Educativa, anexo XXV.

Fernandes-Sisto, F., Marín-Rueda, F. J., \& Urquijo, S. (2010). Relación entre los constructos autocontrol y autoconcepto en niños y jóvenes. Liberabit, 16(2), 217-226. https://bit.ly/382sDoY

Fitch, J. (1935). Vocational guidance in action. Columbia University Press. 
Fryer, D. (1931). The measurement of interests. Enry Holt.

Gallardo-Gallardo, E., Thunnissen, M., \& Scullion, H. (2019). Talent management: context matters. International Journal of Human Resource Management, 31(4), 457-473.

https://doi.org/10.1080/09585192.2019.1642645

García-Cruz, A. (2016). Familia, educación y la construcción de la identidad y del autoconcepto en niños escolares. PsicoEducativa: reflexiones y propuestas, 2(3), 40-46. https://bit.ly/382WbCK

Gil Beltrán J. M. (2005). Prácticas de orientación y asesoramiento. Universitat Jaume I.

Ginzberg, E., Ginsburg, S. Axelrad, S., \& Herma, J. (1951). Occupational Choice An Approach to Theory. Columbia University Press.

González Maura, V. (2004). La orientación profesional universitaria. Una estrategia educativa para el desarrollo profesional responsable. Alertes Psicopedagogía.

Grove, J. M. (1983). Self-concept in vocational choice. Council for Scientific - an Industrial Research Reports, 220-365.

Gruber, H. (1974). Creativity, Psychology and the History of Science. Springer.

Haaland, V. F., Rege, M., Telle, K., \& Votruba, M. (2018). The intergenerational transfer of the employment gender gap. Labour Economics, 52, 132-146. https://doi.org/10.1016/j.labeco.2018.04.004

Hadamard, J. (1945). The Psycology of invention in fhe matbematical. Princeton University Press.

Hayes, A. R., Bigler, R. S., \& Weisgram, E. S. (2018). Of men and money: Characteristics of occupations that affect the gender differentiation of children's occupational interest. Sex Roles, 78, 775-778. https://doi.org/10.1007/s11199-017-0846-8

Holland, J. L. (1976). Vocational preferences. En M. D. Dunnette (Eds.), Handbook of Industrial and organizational Psycholgy (pp. 521-570). Rand-McNally.

Koestler, A. (1964). The act of creation. Macmillan.

Kohler, W. (1969). The task of Gestalt psychology. Princeton University Press.

Kris, E. (1952). Psychoanalytic Exporations in Art. International Universities Press.

Kubie, L. S. (1956). Psychoanlysis and marriage. In V. W. Eisentein (Ed.), Neurotic interaction in marriage (pp. 10-43). Basic Books.

Latiesa, M. (1986). Demanda de Educación Superior y rendimiento académico en la universidad. Ministerio de Educación y Ciencia.

Marín, M., Infante, E., \& Toyano, Y. (2000). El fracaso académico en la Universidad: aspectos motivacionales e intereses profesionales. Revista Latinoamericana de psicología, 32(3), 505 - 517.

https://hdl.handle.net/11441/73318

Maslow, A. (1983). La personalidad creadora. Kairos.

Mednick, S. A. (1962). The associative basis of the creative process. Psychological Review, 69, 220-232. https://doi.org/10.1037/h0048850

Molina, S., \& Fernández, C. (2007). La formación de la orientación profesional como ámbito de intervención en la ciudad. Revista de Ciencias de la Educación, 211, 319-339. http://hdl.handle.net/11162/36279 
Pérez, J. C. N. (1993). El autoconcepto: características estructurales, diferencias evolutivas inter e intraindividuales y su relación con el rendimiento en alumnos de 6 a 11 años [Tesis Doctoral]. Universidad de Oviedo.

Ponsa-Díez, J. (1998). El autoconcepto en la infancia y adolescencia, y los agentes primarios de socialización. Informació Psicológica, 66, 40-50. https://bit.ly/31k9PQL

Riart, J. (2001). La orientación vocacional professional y ocupacional en el Segundo ciclo de la ESO. Educaweb.com

Rivas, F. (1990). La elección de estudios universitarios. Consejo de Universidades.

Rivas, F. (2007). ¿La conducta y asesoramiento vocacional en el mundo de hoy? Revista electrónica de Investigación Psicoeducativa, 5(11) , 5-14. https://doi.org/10.25115/ejrep.v5i11.1233

Rizo-García, M. (2006). La interacción y la comunicación desde los enfoquesde la psicología social y la sociología fenomenológica. Breve exploración teórica. Anàlisi, 33, 45-62. https://bit.ly/34KovZr

Rocabert, E. (1987). La opción universitaria. Un sistema de exploración de la conducta motivacional basado en los intereses profesionales [Tesis Doctoral]. Universidad de Valencia.

Rogers, C. R. (1959). Toward a theory of creativity. En H. H. Anderson (Ed.), Creativity and its cultivation (pp. 6982). Harper \& Brothers.

Santana-Vega, L. E. (1990). La orientación desde las perspectivas psicométrica, clínico-médica y humanista. Qurriculum, 1, 79-92. https://bit.ly/36VIO98

Solbes-Canales, I., Valverde-Montesino, S., \& Herranz-Hernández, P. (2020). Socialization of Gender Stereotypes Related to Attributes and Professions Among Young Spanish School-Aged Children. Frontiers in Psychology,11, e609. https://doi.org/10.3389/fpsyg.2020.00609

Soprano, G. (2013). Ser militar en la Argentina del siglo XXI: Entre una vocación, una profesión y una ocupación. Avá. Revista de Antropología, 23, 71-95. https://bit.ly/3nlhGRO

Super, D. (1967). Psicología de los intereses profesionales. Kapellusz.

Ternera, L. A. C. (2014). El desarrollo del autoconcepto en niños y niñas y su relación con la interacción social en la infancia. Psicogente, 17(31), 67-79. https://doi.org/10.17081/psico.17.31.1470

Tintaya-Condori, P. (2016). Orientación profesional y satisfacción vocacional. Revista de Psicología, 15, 45-58. https://bit.ly/2SLIG3y

Turner, J. D., \& Albro, J. (2017). When I grow up: assessing American children's perspectives on college and career readiness through drawings. Literacy, 51(2). 94-103. https://doi.org/10.1111/lit.12113

Vence, D., Palomares, L., \& Sánchez, A. (2000). Efectos de la identidad social de género sobre las preferencias profesionales. En E. Marchena-Consejero \& C. Alcalde-Cuevas (Coords.), La perspectiva de la educación en el siglo que empieza: Actas IX Congreso INFAD Infancia y Adolescencia, II (pp. 731-735). Universidad de Cádiz.

Wallach, M. A. (1970). Creativity. En P. H. Mussen (Ed.), Carmichael's manual of child psychology (pp. 12111272). Wiley.

Wertheimer, M. (1959). Productive thinking (enlarged ed.). Harper \& Brothers.

Zabala, A. F., \& Palacios, E. G. (2008). El autoconcepto infantil: una revisión necesaria. International Journal of Developmental and Educational Psychology, 2(1), 13-22. https://bit.ly/2ZhehND Esta obra está bajo una Licencia Creative Commons
Attribución-NoCommercial 4.0 International

(cc) BY-NC 